\title{
BMJ Open Quality Promoting subglottic secretion drainage: a quality improvement project in a UK critical care unit
}

\author{
Nicholas Weston Smith, ${ }^{1}$ Michael Spivey ${ }^{2}$
}

To cite: Weston Smith N, Spivey M. Promoting subglottic secretion drainage: a quality improvement project in a UK critical care unit. BMJ Open Quality 2021;10:e001269. doi:10.1136/ bmjoq-2020-001269

Received 13 November 2020 Accepted 18 May 2021
Check for updates

(C) Author(s) (or their employer(s)) 2021. Re-use permitted under CC BY-NC. No commercial re-use. See rights and permissions. Published by BMJ.

${ }^{1}$ Department of Anaesthesia, Royal Cornwall Hospitals NHS Trust, Truro, UK

${ }^{2}$ Department of Critical Care, Royal Cornwall Hospitals NHS Trust, Truro, UK

Correspondence to Dr Nicholas Weston Smith nwestonsmith@nhs.net

\section{ABSTRACT}

The aim of the study was to promote the practice of subglottic secretion drainage (SSD) in a UK critical care unit. SSD is a technique employed to reduce microaspiration of oropharyngeal secretions in patients with cuffed endotracheal airways. Aspiration of oropharyngeal secretions is the accepted cause of the majority of ventilator-associated pneumonia (VAP), a complication of invasive ventilation with high associated mortality. The plan-do-study-act methodology was employed. The local critical care patient database was searched for patients requiring mechanical ventilation via a tracheostomy tube with subglottic port in the 3 months prior to intervention. Patient records were interrogated for evidence of the practice of SSD. The intervention involved the introduction of a tracheostomy care bundle to be prescribed on insertion of a tracheostomy on the critical care unit, in combination with departmental teaching. The bundle included prompts for nursing staff to practise regular SSD and to complete a tracheostomy care plan at the end of shift. A total of 24 patients were included. A review of practice was conducted every 3 months for 1 year. This showed an improvement in documented evidence of SSD from $0 \%$ of days at baseline to $85.7 \%$ of days at 1 year. Implementation of a tracheostomy order set prescribing regular SSD resulted in an improvement in the practice of SSD in patients ventilated via tracheostomy. This has implications for patient outcomes and healthcare costs, given that SSD has been shown to reduce incidence of VAP.

\section{PROBLEM}

The Royal Cornwall Hospitals (RCH) NHS Trust is the major provider of acute care in Cornwall, catering to a population of around 430000 . There were 986 critical care admissions in 2019, 35 of whom had tracheostomies at some point during their critical care stay.

Subglottic secretion drainage (SSD) is a practice designed to reduce the incidence of ventilator-associated pneumonia (VAP) through preventing microaspiration of oropharyngeal secretions. Specific oral endotracheal and tracheostomy tubes are available with subglottic ports to enable regular SSD. The RCH critical care unit uses tracheostomy tubes with subglottic ports as standard.
The focus of the project was to promote the use of SSD in those patients ventilated via a tracheostomy. We aimed to achieve a practice of SSD every 6 hours in all patients ventilated via a tracheostomy on the critical care unit within a year of commencing the project.

Previous local attempts had been made to reduce VAP incidence. Monitoring cuff pressure and maintaining head of bed elevation had become staples of local practice through their inclusion in checklists as part of daily medical reviews. However, the practice of SSD at RCH was not routine on commencing the project. Indeed, baseline data demonstrated no evidence of regular SSD in those patients ventilated via tracheostomy in the 3 months prior to commencing the project. This presented an area for quality improvement, given the evidence that regular SSD reduces rates of VAP. ${ }^{1}$

A quality improvement project using plando-study-act (PDSA) cycles was thought to be an appropriate method for promoting the practice. A stepwise approach would enable the team to evaluate the impact of changes. Further modifications could then be made to maximise the effect and sustainability of the intervention.

\section{BACKGROUND}

VAP is a pneumonia occurring more than 48 hours after endotracheal intubation. ${ }^{2}$ VAP is the most common form of nosocomial infection on critical care units, with a rate of 13.6 per 1000 ventilator days. ${ }^{3}{ }^{4}$ It has an associated mortality between $10 \%$ and $50 \%$, and is associated with significant morbidity, critical care length of stay, antimicrobial use and healthcare costs. ${ }^{15-7}$

The accepted pathophysiology of VAP is microaspiration of oropharyngeal and gastric secretions. ${ }^{8}$ The presence of an endotracheal or tracheostomy tube interferes with normal airway protection mechanisms including swallowing and coughing and may increase oropharyngeal secretions through irritation 
of the mucosa. Treatment of VAP remains a challenge, not least due to high rates of antimicrobial resistance within the critical care population, and therefore there is a focus on prevention. ${ }^{2}$ Several preventative strategies have been developed to reduce VAP incidence. SSD is one such strategy that works by draining pooled secretions from above the cuff of the endotracheal or tracheostomy tube, reducing the risk of microaspiration.

SSD has been the subject of significant research. In the past decade, three meta-analyses have been published in an attempt to understand the potential benefits of SSD. The first, published in 2011, described a risk ratio for VAP of 0.55 (95\% CI 0.46 to $0.66, \mathrm{p}<0.00001$ ) with SSD, as well as reductions in critical care length of stay and duration of mechanical ventilation. ${ }^{9}$ The second, published in 2016, also demonstrated a significant risk ratio for VAP of 0.58 with SSD but did not establish significant reductions in critical care or hospital length of stay, duration of mechanical ventilation or mortality. ${ }^{1}$ More recently in 2020, a third meta-analysis again demonstrated a significant reduction in VAP with SSD, as well as a reduction in mortality. ${ }^{10}$

The use of oral endotracheal and tracheostomy tubes with SSD ports has been incorporated into national guidance in the USA for those patients expected to require greater than 48 hours of mechanical ventilation-the time frame over which a VAP develops. ${ }^{7}$ Locally, all tracheostomies inserted on the RCH critical care unit have a subglottic port to enable SSD as it is anticipated that the majority of this population will require greater than 48 hours of mechanical ventilation via their tracheostomy.

In addition to SSD, other evidence-based measures for reducing the incidence of VAP include ${ }^{7}$

- Maintaining endotracheal cuff pressures.

- Head of bed elevation to $30^{\circ}-45^{\circ}$.

- Minimising sedation and muscle relaxant.

- Minimising duration of mechanical ventilation.

- Selective oral and gastric decontamination.

Many centres have successfully implemented care bundles combining a selection of these strategies, demonstrating a subsequent reduction in their incidence of VAP. ${ }^{11}{ }^{12}$ Locally, it was decided to first focus on promoting regular SSD before considering its inclusion within a VAP prevention bundle.

\section{MEASUREMENT}

\section{Population}

The RCH critical care unit uses the Philips Medical Systems CareVue clinical information system. This system allows for electronic documentation and prescription, and enabled identification of all patients that received mechanical ventilation via a tracheostomy at RCH by the local data collector. The following exclusion criteria were applied as these patients would not routinely have a tracheostomy with SSD port inserted:

- Those patients admitted to the critical care unit with existing long-term tracheostomies.
- Those patients admitted to the critical care unit with a tracheostomy following head and neck surgery.

- Those patients repatriated to the critical care unit from other centres.

\section{Outcome}

The aim of the project was to promote regular SSD. The major outcome measured would therefore be the number of days on which regular SSD every 6 hours occurred as a proportion of the number of days ventilated via a tracheostomy. Once patients were identified, the following data were collected through a review of the electronic patient notes within CareVue by the authors:

- The number of days ventilated via the tracheostomy (ventilator days).

- The number of ventilator days on which regular SSD was documented.

If there was documentation of SSD at a minimum of every 6 hours within a 24-hour period, then this was included as evidence of regular SSD. If this requirement was not met within a 24-hour period, then regular SSD was deemed not to have occurred on this day. The number of ventilator days on which SSD occurred as a percentage of total ventilator days would provide the outcome measure of SSD compliance.

\section{Baseline measurement}

The baseline measurement identified nine patients during the 3 month period of November 2018 to January 2019. Interrogating the electronic patient notes within CareVue revealed that these nine patients combined for 111 ventilator days.

\section{Ongoing measurement}

It was decided that the team would repeat the cycle of data collection every 3 months. This would enable sufficient time for interventions to be introduced and modified between cycles while providing a manageable study population for each cycle. At each stage, suitable patients were identified, and exclusion criteria were applied as stated earlier. The electronic notes were then reviewed by authors for evidence of regular SSD.

\section{DESIGN}

A key component of the design was to incorporate the intervention into the CareVue information technology system. All documentation and prescribing on the RCH critical care unit are done on CareVue, and any change had to fit with current CareVue practices. It was expected that by using existing IT systems to implement change, the outcomes would be more likely to become accepted practice, and therefore any improvements more likely to be sustainable.

The initial intervention was the development of a prescription for SSD every 6 hours within CareVue. The functionality of CareVue means that when an item is prescribed, nursing staff are reminded to carry out the prescription at the allocated time. Once the prescription 
is carried out, nursing staff document that it has been done via checking a box. By making use of the prescribing functionality of CareVue, the team would be adapting an existing process proven to be reliable for the prescription of medications, to meet the aim of ensuring regular SSD.

The prescription was incorporated into a new "care bundle' to further promote tracheostomy nursing care. This care bundle, once prescribed, would include the prescription for SSD every 6 hours, a reminder for nursing staff to complete a tracheostomy care plan every 12 hours within their shift (an existing CareVue document), and a reminder for clinicians to consider a change of tracheostomy at 90 days.

The quality improvement team included a trainee doctor, a staff nurse and an intensive care consultant. Inclusion of a staff nurse was important in promoting the project among nursing staff, the group that would be taking on the extra work of increased SSD. The project required working closely with local IT staff to design and implement effective changes within the CareVue system. Working as a multidisciplinary team would help to provide a good understanding of the processes involved in both prescribing and carrying out prescriptions on the critical care unit.

Patients and the public were not involved in the design, conduct or reporting of the quality improvement project.

\section{STRATEGY}

\section{PDSA cycle 1}

The care bundle was implemented in February 2019 following a baseline measurement that demonstrated no evidence of regular SSD. The intervention resulted in a moderate improvement in SSD compliance. Results following the first cycle were presented at a departmental governance meeting, and this was an opportunity to promote the project, as well as the benefits of SSD.

While results following the first cycle were encouraging, the intervention had not achieved the stated aim of regular SSD for all patients ventilated via a tracheostomy. Further changes would therefore be needed. It was noted that when the care bundle had been prescribed, compliance with SSD targets was good; however, the bundle had only been prescribed in two of four patients. It became clear that the initial intervention relied heavily on the assumption that medical staff knew about the existence of, and would remember to prescribe, the bundle for appropriate patients.

\section{PDSA cycle 2}

The next stage was to focus attention on ensuring that the care bundle was being prescribed. The existing tracheostomy insertion documentation on CareVue was modified to include a tick-box reminder to prescribe the tracheostomy care bundle. This would prompt the clinician responsible for inserting the tracheostomy to prescribe the care bundle immediately after insertion. It was anticipated that this prompt would ensure that the care bundle was prescribed for every patient who had a tracheostomy inserted on the unit. The next round of data collection demonstrated a further improvement over the subsequent 3 months. Again, results were presented at the departmental governance meeting, providing a further opportunity to remind clinicians to prescribe the care bundle.

\section{PDSA cycle 3}

A further 3 months of data collection demonstrated a consistent increase in the occurrence of regular SSD. Interestingly, further analysis of the data demonstrated that over the previous two rounds of data collection, when the care bundle had been prescribed, compliance with regular SSD remained high.

\section{RESULTS}

Over the course of 1 year, 24 patients were included in the data for a total of 353 ventilator days. Rates of SSD compliance as the number of days on which regular SSD occurred as a proportion of the total number ventilator days increased throughout the study period (table 1).

At baseline, there was no documented evidence of regular SSD. By 3 months, this had improved, and the last 6 months of data collection demonstrated a sustained improvement in the regularity of SSD. Results are displayed with respect to interventions in a run chart (figure 1).

It was noted through the PDSA cycles that when the care bundle had been prescribed, compliance with regular SSD was good. On the other hand, in cases where the care bundle had not been prescribed, there were no days on which regular SSD occurred (table 2).

Table 1 Number of days ventilated via tracheostomy, number of days ventilated with regular SSD and percentage of days ventilated with regular SSD within each 3-month cycle

\begin{tabular}{lcccc}
\hline & Baseline & PDSA cycle 1 & PDSA cycle 2 & PDSA cycle 3 \\
\hline Number of patients & 9 & 4 & 8 & 3 \\
Ventilator days & 111 & 65 & 142 & 35 \\
Number of ventilator days with regular SSD & 0 & 20 & 120 & 30 \\
SSD compliance (\%) & 0 & 30.8 & 84.5 & 85.7 \\
\hline
\end{tabular}

PDSA, plan-do-study-act; SSD, subglottic secretion drainage. 


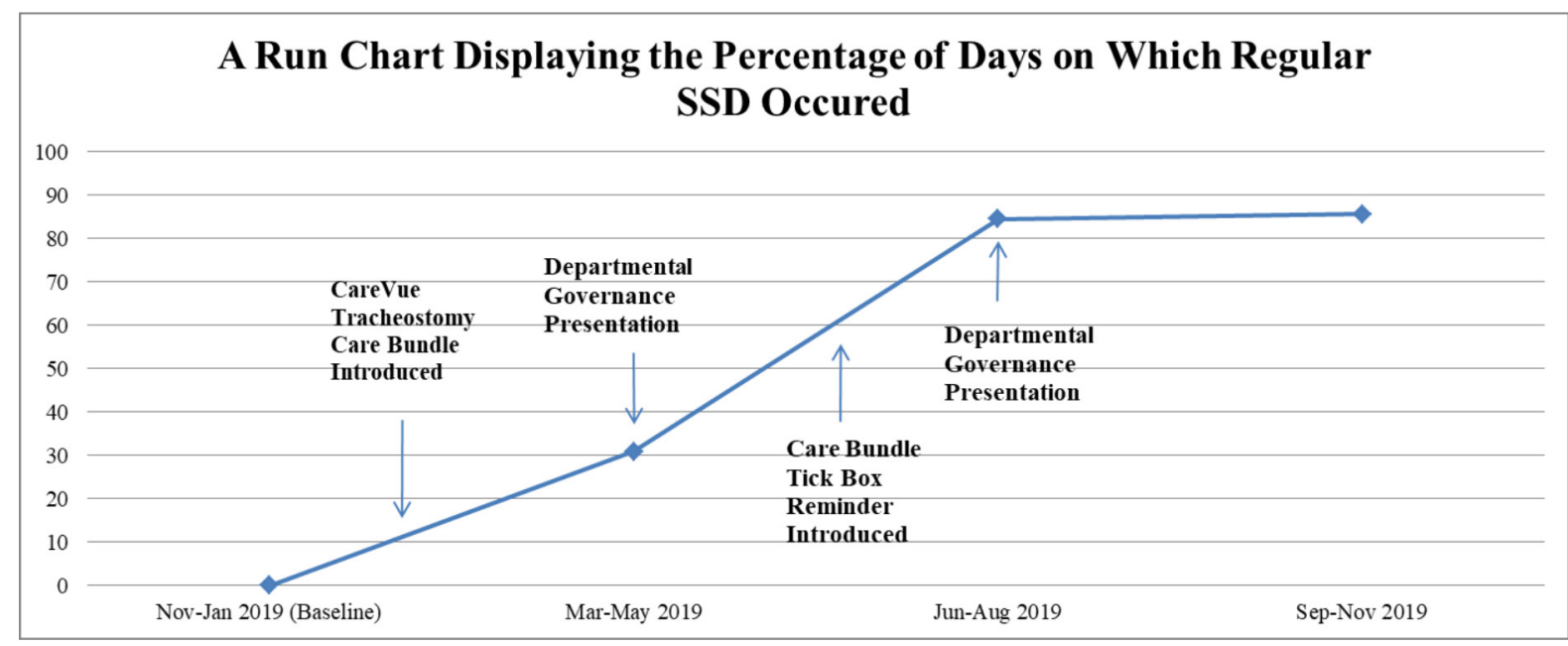

Figure 1 Run chart displaying the percentage of days on which regular SSD occurred with reference to the timeline of changes introduced throughout the project. SSD, subglottic secretion drainage.

CareVue contains all documentation of nursing and medical care on the unit. Owing to consistently excellent documentation by nursing staff, there were minimal missing data. When SSD was not done, the reason for this was described within CareVue. If the reason was thought to be adequate (eg, absence from the unit), then this data point was included as an episode of SSD occurring. If there was no reason, or an inadequate reason documented, then this was included in the results as regular SSD not occurring.

\section{LESSONS AND LIMITATIONS}

Lessons were learnt throughout the process and the project had its strengths and limitations. Using the functionality of an IT system to implement quality improvement interventions, while incorporating the expertise of a multidisciplinary team meant that the changes introduced combined well with current practice. However, smaller than expected patient numbers and an uncertainty over whether the measured change reflects an improvement in documentation or a real change in practice are significant drawbacks.

The major strength of this project was the ability to work within an integrated IT system. Using an existing system meant no extra training was required for staff carrying out the intervention or collecting the data. There were no additional forms or stickers, reducing the burden of the intervention for nursing staff. Being able to use the functionality of CareVue to introduce a prescription for SSD helped to ensure good compliance with the intervention from nursing staff.
A further strength of the project was the multidisciplinary nature of the team, as well the availability and expertise of local IT staff. The member of staff responsible for making changes within CareVue was himself a staff nurse and knew the system's strengths and limitations. Having medical and nursing staff involved in designing the intervention meant that there was a good understanding behind the processes involved with prescribing, carrying out prescriptions and documenting care on the unit. This resulted in an intervention that was reliable and sustainable.

Collecting a second round of data after 3 months and analysing the results helped the team to modify the intervention as the project was progressing. It was noted at this stage that the care bundle when prescribed was leading to excellent documentation of SSD; however, it had not been prescribed in half of the patients, leading to only $30.8 \%$ compliance with regular SSD. Redesigning the process to promote care bundle prescription was a key step in maximising the effect of the intervention at an early stage. Waiting a year to reaudit would have resulted in time wasted.

An added benefit of an online system was the ease with which records can be interrogated, allowing for quick and complete data collection. Easy data collection makes quality improvement more accessible, and projects are more likely to be completed when full data are readily available.

The project was not without its limitations. It is difficult to determine whether the improvement in regularity of SSD was really a change in practice or simply the result

\begin{tabular}{|c|c|c|c|c|c|c|}
\hline \multirow[b]{2}{*}{ Care bundle prescribed } & \multicolumn{2}{|c|}{ PDSA cycle 1} & \multicolumn{2}{|c|}{ PDSA cycle 2} & \multicolumn{2}{|c|}{ PDSA cycle 3} \\
\hline & $\mathrm{y}$ & $\mathrm{n}$ & $\mathrm{y}$ & $\mathrm{n}$ & $\mathrm{y}$ & $n$ \\
\hline
\end{tabular}

n, no; PDSA, plan-do-study-act; SSD, subglottic secretion drainage; y, yes. 
of more complete documentation. At baseline, documentation of SSD was very sporadic and there were no days on which any patient had four episodes or SSD every 6 hours documented. However, at this time, there was no dedicated location within CareVue to document SSD if it was carried out. The intervention provided a reminder to carry out SSD but also a place in which staff could document that it was being done. Whether the results simply reflect better documentation or indeed better practice is unclear. A way around this issue would have been to survey nursing staff at baseline to get a measure of whether they were doing regular SSD in appropriate patients or not.

Over the course of the last 6 months of data collection, two patients were not prescribed the care bundle. Both were patients who had their tracheostomy with SSD port inserted in theatre on behalf of the critical care unit, bypassing the tick-box reminder included in the tracheostomy insertion checklist. The tracheostomy care bundle was not prescribed and neither patient had any documented evidence of SSD as a result. This was an unanticipated failure of the design of the intervention, resulting in a cohort of patients bypassing the reminders put in place. This should form a focus for further quality improvement work.

Finally, patient numbers were lower than expected. The initial baseline period of 3 months of data collection identified nine patients for a combined 111 days. Subsequently, it was determined that 3 monthly intervals should provide reasonable patient numbers. Unfortunately, the subsequent 3 months included only four patients (65 ventilator days), and the final 3 months of data collection included three patients (35 ventilator days). If a single patient in these cohorts was not prescribed the care bundle, then it led to major changes to the results. Conversely, having small patient numbers did allow us to analyse each patient's records in detail. This helped us to determine the reasons why the bundle had not been prescribed or why SSD had not happened when prescribed.

\section{CONCLUSION}

Our aim was to achieve regular SSD every 6 hours in all patients ventilated via a tracheostomy with dedicated subglottic port inserted at RCH. After 1 year, we have demonstrated an improvement in the documentation of SSD every 6 hours from $0 \%$ to $85.7 \%$ of days. While this falls below our $100 \%$ target, it clearly represents an improvement. The reasons for falling below our target were not catching those patients that had a tracheostomy with SSD port inserted in theatre within the intervention, and this cohort should be the focus of further quality improvement.

The cost of a VAP in a UK critical care unit has been calculated in the region of $£ 9000$, while studies in the USA have calculated costs between $\$ 10000$ and $\$ 40000 .^{13-15}$ Cost analyses have shown that implementing regular SSD is a cost effective strategy, in spite of the additional cost of the subglottic port endotracheal tube. ${ }^{16-18}$ Meta-analysis has given a number needed to treat of 11 for SSD. ${ }^{9}$ Our project did not involve any additional material costs given tracheostomies with subglottic ports were already being used at the RCH critical care unit. Assuming similar patient numbers to the study year $(n=24)$, if our project creates a sustained improvement in SSD with $85 \%$ compliance over the coming year, we would expect to prevent around two cases of VAP and its associated economic burden.

The link between regular SSD and reduced rates of VAP is well documented. Given that the methodology described by this project has been shown to promote regular SSD, it would be interesting to note whether it has impacted on local rates of VAP. In order to evaluate this, controlled study would be required, the intervention arm of which could employ the processes described within this report. While the project focused on those patients with tracheostomies, there is scope for expansion to include patients ventilated on critical care via oral endotracheal tubes. Although not used at RCH currently, oral endotracheal tubes are available with subglottic ports for use when prolonged mechanical ventilation is anticipated. It would be interesting to note how centres that employ the use of oral endotracheal tubes with subglottic ports have gone about creating a culture of regular SSD. Indeed, units employing these endotracheal tubes may find this methodology of promoting SSD helpful.

Finally, all quality improvement work should be sustainable. While junior doctors rotate, nursing staff are often employed on a longer-term basis. The project will be ongoing and led by nursing staff with the aim of creating a sustained improvement in patient care and outcomes.

Acknowledgements Laura Muir- Critcal Care Staff Nurse, RCH Critical Care Unit; Simon Caddel- CareVue Lead Nurse, RCH Critical Care Unit; John Anderson- Data Collector, RCH Critical Care Unit.

Contributors NWS: guarantor; responsible for the conception of the project, the design of the interventions, data collection and analysis; and primary author. MS: responsible for the conception of the project, drafting of the report and approval of the version to be published.

Funding The authors have not declared a specific grant for this research from any funding agency in the public, commercial or not-for-profit sectors.

Competing interests None declared.

Patient and public involvement Patients and/or the public were not involved in the design, conduct, reporting or dissemination plans of this research.

Patient consent for publication Not required.

Provenance and peer review Not commissioned; externally peer reviewed.

Data availability statement All data relevant to the study are included in the article.

Open access This is an open access article distributed in accordance with the Creative Commons Attribution Non Commercial (CC BY-NC 4.0) license, which permits others to distribute, remix, adapt, build upon this work non-commercially, and license their derivative works on different terms, provided the original work is properly cited, appropriate credit is given, any changes made indicated, and the use is non-commercial. See: http://creativecommons.org/licenses/by-nc/4.0/. 


\section{REFERENCES}

1 Caroff DA, Li L, Muscedere J, et al. Subglottic secretion drainage and objective outcomes: a systematic review and meta-analysis. Crit Care Med 2016;44:830-40.

2 Kalil AC, Metersky ML, Klompas M, et al. Management of adults with hospital-acquired and ventilator-associated pneumonia: 2016 clinical practice guidelines by the infectious diseases Society of America and the American thoracic Society. Clin Infect Dis 2016;63:e61-111.

3 Vincent JL, Bihari DJ, Suter PM, et al. The prevalence of nosocomial infection in intensive care units in Europe. Results of the European prevalence of infection in intensive care (EPIC) study. EPIC international Advisory Committee. JAMA 1995;274:639-44.

4 Charles MP, Kali A, Easow JM, et al. Ventilator-Associated pneumonia. Australas Med J 2014;7:334-44.

5 Choudhuri AH. Ventilator-Associated pneumonia: when to hold the breath? Int J Crit IIIn Inj Sci 2013;3:169-74.

6 Koenig SM, Truwit JD. Ventilator-Associated pneumonia: diagnosis, treatment, and prevention.. Clin Microbiol Rev 2006;19:637-57.

7 Klompas M, Branson R, Eichenwald EC, et al. Strategies to prevent ventilator-associated pneumonia in acute care hospitals: 2014 update. Infect Control Hosp Epidemiol 2014;35:915-36.

8 Estes RJ, Meduri GU. The pathogenesis of ventilator-associated pneumonia: I. mechanisms of bacterial transcolonization and airway inoculation. Intensive Care Med 1995;21:365-83.

9 Muscedere J, Rewa O, McKechnie K, et al. Subglottic secretion drainage for the prevention of ventilator-associated pneumonia: a systematic review and meta-analysis. Crit Care Med 2011;39:1985-91.

10 Pozuelo-Carrascosa DP, Herráiz-Adillo Ángel, Alvarez-Bueno C, et al. Subglottic secretion drainage for preventing ventilator-associated pneumonia: an overview of systematic reviews and an updated meta-analysis. Eur Respir Rev 2020;29:190107.

11 Daniel M, Booth M, Ellis K, et al. Details behind the dots: how different intensive care units used common and contrasting methods to prevent ventilator associated pneumonia. BMJ Qual Improv Rep 2015;4. doi:10.1136/bmjquality.u207660.w3069. [Epub ahead of print: 1203 2015].

12 Marini AL, Khan R, Mundekkadan S. Multifaceted bundle interventions shown effective in reducing VAP rates in our multidisciplinary ICUs. BMJ Qual Improv Rep 2016;5. doi:10.1136/ bmjquality.u205566.w2278. [Epub ahead of print: 0404 2016].

13 Luckraz H, Manga Na'ngono, Senanayake EL, et al. Cost of treating ventilator-associated pneumonia post cardiac surgery in the National health service: results from a propensity-matched cohort study. $J$ Intensive Care Soc 2018;19:94-100.

14 Restrepo MI, Anzueto A, Arroliga AC, et al. Economic burden of ventilator-associated pneumonia based on total resource utilization. Infect Control Hosp Epidemiol 2010;31:509-15.

15 Kollef $\mathrm{MH}$, Hamilton CW, Ernst FR. Economic impact of ventilatorassociated pneumonia in a large matched cohort. Infect Control Hosp Epidemiol 2012;33:250-6.

16 Kelley SD. Number needed to treat for subglottic secretion drainage technology as a ventilator-associated pneumonia prevention strategy. Crit Care 2012;16:446.

17 Hallais C, Merle V, Guitard P-G, et al. Is continuous subglottic suctioning cost-effective for the prevention of ventilator-associated pneumonia? Infect Control Hosp Epidemiol 2011;32:131-5.

18 Branch-Elliman W, Wright SB, Howell MD. Determining the ideal strategy for ventilator-associated pneumonia prevention. Costbenefit analysis. Am J Respir Crit Care Med 2015;192:57-63. 